\title{
A Web-based Database System for Providing Technical Information on ATM Networking Platforms
}

\author{
Kimon Kontovasilis*, George Kormentzas*, Anastasios Kourtis*, \\ Nikolas Mitrou**, John Soldatos** and Evangelos Vayias** \\ * National Center for Scientific Research "DEMOKRITOS", Institute for Informatics \& Telecommunications, Greece \\ **National Technical University of Athens, Electrical \& Computer Eng. Dept., Computer Science Division, Greece
}

\begin{abstract}
This paper presents a Web-based database hosting technical information about pioneering ATM networking platforms, associated research activities engaging these platforms, and related important trials conducted in the framework of these research activities. The paper outlines the organisation and structure of the information content in the database and discusses methods of access through the WWW interface. Besides the "static" information offered by the database, other Java-based tools provide for the on-line monitoring of the status of the ATM platforms and for manipulating data arising from technological trials on these platforms. The integration of these tools with the database, under a common WWW interface is discussed.
\end{abstract}

Keywords: ATM networks, WWW, information systems, Java-based tools, remote monitoring, trials.

\section{Introduction}

The ACTS (Advanced Communication Technologies and Services) research programme has been the primary vehicle for research on advanced telecommunication systems, conducted within the EU framework. During the lifetime of ACTS, many relevant research projects maintained means for electronic dissemination of technical information pertaining to these projects. There were even projects, like InfoWin (The Multimedia Information Window for ACTS - AC113) (see InfoWin Proj., 1996) that focused specifically on providing means for facilitating information dissemination. In this paper, we focus on the Web-based information system developed by the WATT (WWW Windows for ACTS Trials and Testbeds - AC235) research project (see WATT Proj., 1996), in which the authors have participated. The aim of the WATT system is rather different from that of other projects oriented towards information dissemination. Instead of dealing with the technical results of specific projects, WATT targets technical information relating to the European prototype ATM networking platforms. These network islands play a key role in the broadband telecommunications research undertaken in Europe. They reflect the culmination of massive technical work undertaken over the years, from the RACE (Research and development in Advanced Communication in Europe) to the ACTS framework. During the course of this work, the islands have been built, modified and supplemented, so as to be representative of the technological state of the art.

Relieved from the restrictions that necessarily accompany environments targeted to "production" work, these prototype networks are particularly suitable as experimental testbeds (the term of choice for referring to them in the vocabulary of the described database) and, in recognition of this fact, they have been directly engaged by many research projects in technological experiments (also called trials) demonstrating technical results. Thus, the said testbeds have become the focus of important technolo-

\footnotetext{
${ }^{\dagger}$ Work partially funded by the European Union, through the research project AC-235 WATT (WWW Windows for ACTS Trials and Testbeds), in the ACTS (Advanced Communications Technologies and Services) research program.
} 
gical events that illustrate and advance the state of the art of ATM and related technologies. As such, they are also ideal focal points for disseminating this up-to-date technical information to a wider engineering and research community, both within and outside the European Union (Alles, 2000).

The purpose of the system described in the paper, called the "WATT database" (and abbreviated throughout as WDB) is twofold:

1. to provide information about important ATM testbeds, thus demonstrating typical paradigms of early comprehensive ATM installations; and

2. to become an information gateway for important technological trials that employ these ATM testbeds.

The first objective has been met through the provision of comprehensive information, mostly in HTML format, about the components and subsystems comprising the testbeds, hierarchically organised and appropriately cross-referenced for effective perusal and retrieval. Note that WDB is not a "classical" database in the sense that no underlying DBMS engine is used. It is the data organization that provides a discipline to the whole system (Ramakrishnan et al, 2002).

While the first objective addresses "static" information (the configuration of the testbeds does not change very frequently), the second objective is of a dynamic flavour, because trials are events, performed once, in a tight timeschedule. As such, trials have an important "live" component and it is desirable that this component be accessible to the largest audience possible. WDB is intended to enable remote audiences to "participate" in important trial events.

Towards this end, trials planned for performance on a testbed covered within WDB are "advertised" in the database, so that potential trialwatchers may be informed of the trial scenario and of the way the testbed is involved. Furthermore, during the actual performance of these trials, users of WDB may engage a Java-based software tool (see WATT Proj., 1997e, Mitrou et al., 1997b and Vayias et al., 1997b) that enables remote monitoring of the testbed's status in a dynamic fashion, as the trial evolves. Finally, after the completion of the trial, the relevant
WWW page in WDB is updated to include a synopsis of the trial results.

For certain important trials, special data files that contain records of the testbed's status during the course of the trial may become available; in this case, they are reachable through access to WDB, from within the WWW pages describing the trials. The data files may be downloaded by the user and subsequently processed, by means of a special software tool (see WATT Proj., 1997c and Vaduva et al., 1997), called the "demonstration authoring tool", locally, at the user's computer, so as to generate a dynamic visualisation of the recorded status changes. Instances of this visualisation may be saved in files and used as supporting material, towards the preparation of lecture notes or reports on the particular technological issues highlighted by the trial.

Both the tools for monitoring trials on-line and those for processing trial results off-line are applications of a client/server software system, called the "WATT Monitoring System" (and abbreviated throughout as WMS), which enables the remote monitoring of the status of a target testbed. The WMS client is Java software, typically invoked from within WDB through an appropriate link. Upon invocation, the WMS client is loaded on top of the user's WWW browser (which must be Java-capable); subsequently the WMS client automatically establishes a connection to the testbed that will be monitored. Once this connection is established, the user may issue monitoring commands, choosing from a set of available operations. A module of the WMS client software, called the "viewer", acts as a graphical user interface that displays a graphical representation of the testbed's topology and handles, through menu selections or mouse clicks, the monitoring requests. These requests are forwarded over the Internet to the server part of WMS, which resides at a computer by the testbed, for processing, then the replies are sent back to the user. Note that WMS requires appropriate support from the testbed's network management system, which must interface to the server part of WMS. Because of this requirement, only some of the testbeds covered by WDB may be monitored remotely. For more details on the WMS architecture see (WATT Proj., 1997a). 
The rest of the paper discusses the structure and features of the "static" component of WDB and the integration of this component with the other Java-tools. Section 2 presents generic features and capabilities that are important from the user's, implementor's, or maintainer's point of view. These features have supplied the motivation for particular design decisions and have served as "checkers" of the appropriateness of the design afterwards. The abovementioned integration issues are mainly discussed in this section. Subsequently, Section 3 outlines the information content and its structure, describing, in particular, the various information categories included in WDB and specific information items for these categories. Section 4 comments on implementation issues and on the present operational status of WDB, while Section 5 presents major trials that have been covered by WDB. Finally, Section 6 recapitulates the paper and provides some concluding remarks.

\section{Generic WDB Features and Capabilities}

WDB was designed with the intention of providing a versatile, convenient to use, and powerful system. In the specific context of ATM networking platforms, these general attributes translate into the following characteristics:

1. Modularity and extensibility;

2. Uniform structure and appearance, across different testbed, project, or trial instances;

3. Comprehensiveness;

4. Easy navigation to the desired information and balanced support for users aiming at different information categories;

5. Provision for the potential of classified access to certain information items;

6. Integration with the WMS client and the demonstration authoring tool.

The first feature is important from the implementor's and maintainer's point of view, especially when taking into account that, besides the ATM testbeds initially covered by the database, additional testbeds, projects, or trials may be incorporated in the future. Extensibility, in particular, is primarily meant for incorporating additional testbeds, not so much incorporating new unanticipated information categories. In the latter case, the design may have to be readjusted, although it is desirable that such adjustments be kept at a moderate level and remain localised.

In the context of the second characteristic, uniformity is meant as similar structuring of the information over different testbeds, despite the expected (in some cases wide) variations in the actual information content. Uniformity is a major factor towards ease of usage, seen both from a psychological and a procedural angle. Furthermore, a homogeneous structure enables easy comparison between various testbeds over a certain aspect, e.g., their switching technology, and thus increases the utility of WDB. A robust and uniform structure is also of paramount importance in enforcing a discipline on the whole system since, as already remarked, no DBMS engine underlies WDB. Finally, note that, besides structure, the notion of uniformity is extended to also imply a certain degree of similarity in the visual appearance of information across different testbeds; this promotes the sense of an integrated environment. Similarity in appearance, however, is not overemphasised or excessively enforced, since it is indeed desirable that each testbed participating in WDB maintains its particular character at this level as well.

The desire for comprehensiveness is self-explanatory. Note that the objective of WDB is to provide information so as to cover not only ATM testbeds, but also associated research projects and important trial results. Thus, projects and trials are important entities to be covered by the database, although their association with a particular ATM testbed is an essential prerequisite for their inclusion. Note that the requirement for comprehensiveness is partly antithetical to the desire for uniformity, due to the fact that testbeds, especially those of an experimental orientation, present wide variations in their scope, configuration and functionality. The best compromise between the two objectives has been sought. Finally, it is to be remarked that comprehensiveness does not necessarily imply that large amounts of information have to be stored in a single place. Under many circumstances, links to information residing elsewhere have been provided; this is fairly easy for a World Wide Web environment (see Chapin et al., 1997). 
User-friendly navigation (Feature 4) is a very important contributor to the utility of WDB, especially if one considers the large amounts and the diversity of information held in the database. Uniformity in structure is very helpful in this respect. Note that user-friendliness should apply equally to different categories of users. For example, there are users that may interrogate the database for some particular project that is active on a testbed, then follow down links and reach trial results in the framework of this project. On the other hand, there may be users directly interested in trial results on some particular technological issue. Users of the second kind should have the possibility to follow a path more natural for their needs, towards the information they are after; in this case, the alternate path may by-pass information on particular project and immediately access a collective pool of trials on the relevant technological issue.

Given the security enforcement facilities present at current WWW servers (Larson et al., 2000), WDB employs some of these facilities for providing the potential of classified access to parts of the database. This enables confidential or copyrighted information items to be kept on the same machine as the publicly available ones. Such an arrangement effectively permits the use of a single information source, namely WDB, by many classes of users with varying accessauthorisation privileges.
The last feature in the list of items above relates to integration with WMS, namely the system that provides for the remote monitoring of testbeds, including the monitoring of the evolution of trials on these testbeds, and the demonstration authoring tool that enables the retrieval and post-processing of files containing trialdata. (This issue is applicable only to testbeds that support WMS through an appropriate interface to the Network Management System.) The integration is feasible because both the user interface to WMS and the demonstration authoring tool are Java-based applications that run on top of ordinary WWW browsers; thus their usage meshes well with the WWW orientation of WDB. Besides being feasible, the integration is also desirable, because is increases the utility of WDB. For example, a user that is about to login, through WMS, to a remote testbed to monitor a particular activity, may before doing that, easily consult the appropriate sections of WDB in order to be briefed on the particular event to be monitored, or to review the architecture and capabilities of the ATM testbed.

\section{Organisation of the Material in the Database}

WDB contains information about ATM testbeds, research projects associated with these testbeds

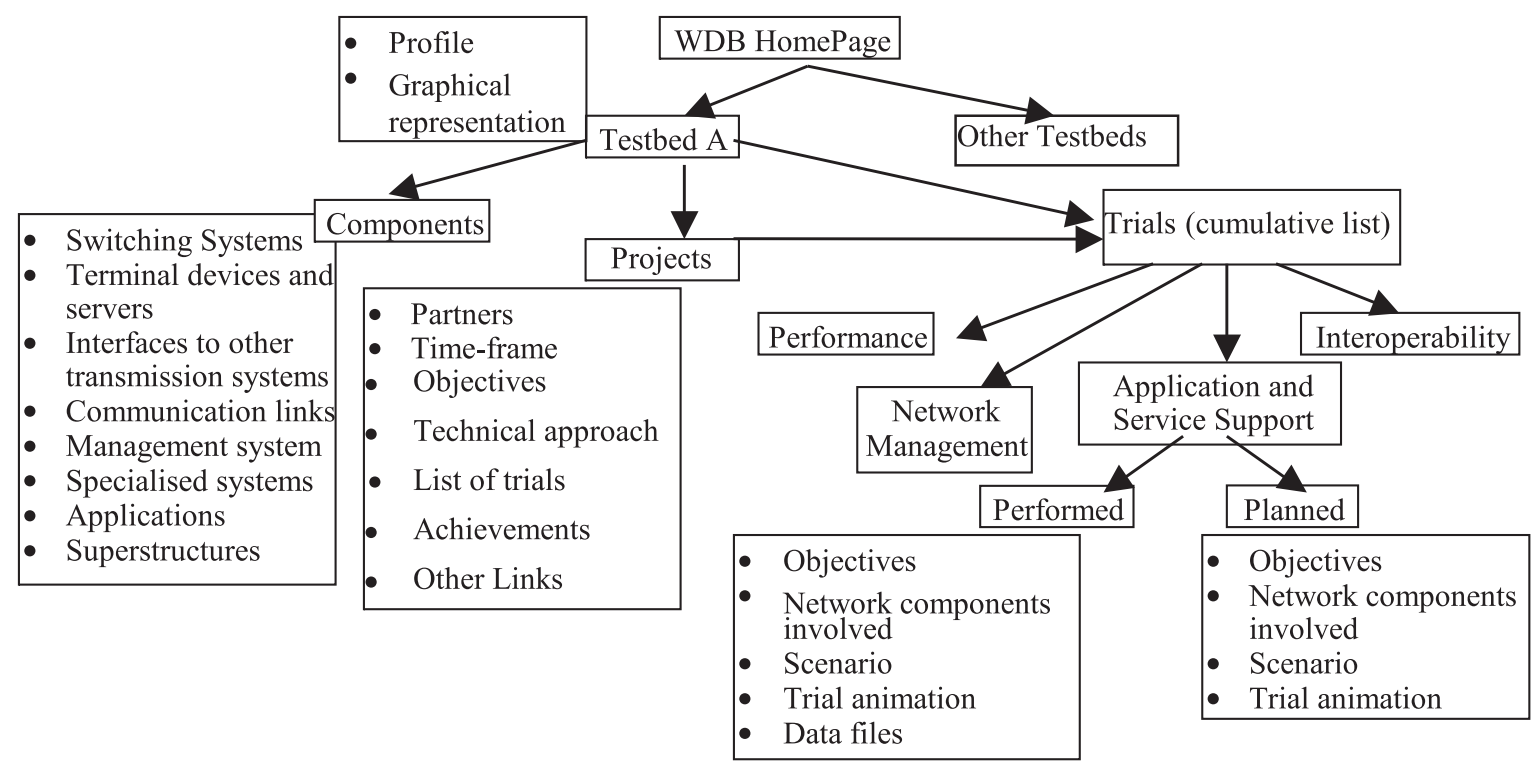

Fig. 1. Outline of the WDB structure. 
and trials scheduled in the context of these research projects. All information relating to a particular testbed, is organised in a "treelike" hierarchy, where there is one tree for each testbed included in the database. The hierarchy is depicted in Figure 1. The "root" of each tree is occupied by a WWW "home" page for the respective testbed that contains general information, including a brief profile of the testbed, and links to other pages, lower in the tree hierarchy, that provide more specialised information.

The information below the "root" level is organised into three major categories:

- Components and subsystems of the testbed: It contains information about the testbed per se, regarded as a network/system. This category is further described in subsection 3.1.

- Research projects: This is a list of the research projects associated with the testbed. The list contains links that lead to individual pages (or sections in a page) describing each project. Details on the information content relating to projects is given in subsection 3.2.

- Trials run on this testbed: This is a list of important trials that have been performed on the testbed, or are scheduled for performance at a later time. Individual trials are further organised according to category and according to status, as it will be explained in subsection 3.3. The link at the "root" page leading to trial-related information points to the top level of this organisation. Note that the trials correspond to research projects that have scheduled the trials as part of their activities. The link at the "root" page leads to a cumulative list of trials from all projects. Access to the subset of the trials that are relevant to a particular project is possible through another link from within the page describing this project.

\subsection{Information About the Components of a Testbed}

This part of WDB includes detailed information about the devices and systems that combine to formulate the testbed. To manage the complexity of current ATM networking platforms, various components are organised into a number of categories, according to function. These categories are:
- Switching systems: It includes devices responsible for switching and multiplexing within the ATM network.

- Terminal devices and servers: It includes components that may be viewed as "endpoints" from the network's point of view. Typical members of this category include computers with a network interface, multimedia terminals and video, multimedia, or database servers.

- Interfaces to other transmission systems: Devices having an interconnection nature go in this category. Typical members include mappers, interworking units, routers and bridges.

- Communication links: It provides a home for the description of the actual media interconnecting the components of the network platform (and connecting this platform to the "outside world").

- Management system: It covers all systems and functions relating to network management.

- Specialised devices: Devices of a somewhat specialised or peripheral nature, with respect to the main networking or application environment. Typical members of this category are: measurement and testing devices, traffic generators and accounting and/or charging units.

- Applications: A repository for systems at an application level that rely on ATM networking facilities and operate on top of them, so as to provide a service, either generalpurpose, or custom. A typical example is a hardware and/or software system interoperating with the network to provide a distributed multimedia service.

- Superstructures: It applies to cases where there is a tight interconnection between the described testbed and other network islands, in such a way that a higher-level entity may be identified. The notion of a superstructure applies only if the individual islands may be regarded as independent interconnected entities. Otherwise, the whole system logically is a single platform and the superstructure category is not relevant. 


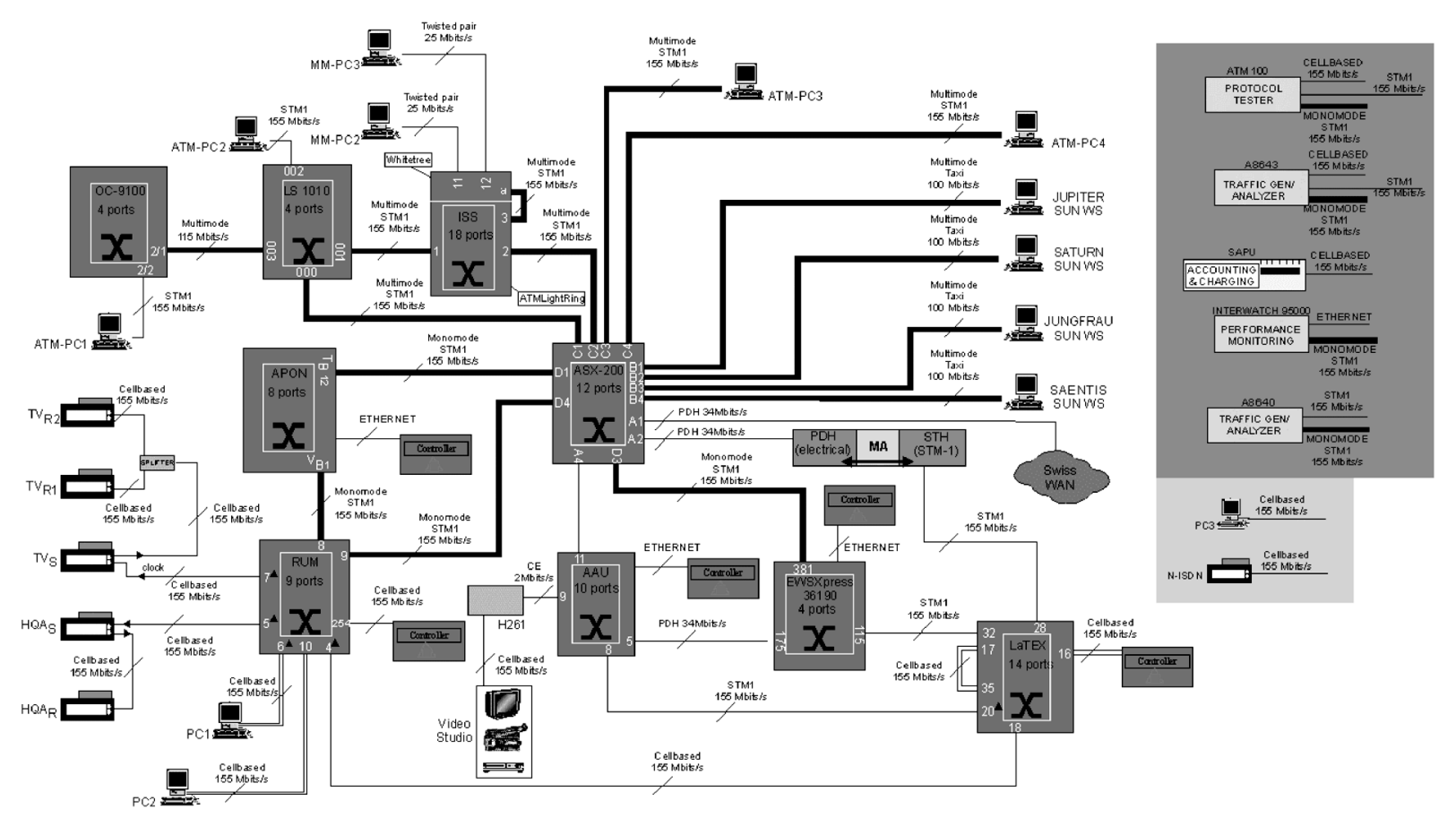

Fig. 2. Graphical representation of a tesbed's topology.

These categories are "exhaustive", in the sense that they are intended to cover all possible cases that may arise in an ATM platform. For an actual testbed without any devices of a specific category, the respective description in WDB omits the irrelevant category altogether (rather than providing an "empty" header). The information for each individual member in the categories above, has been further organized into fully specified information items, suitable for the object under description. Description of a switching device, for example, may include information for:

1. the manufacturer, the relevant product line, the specific model, etc.; a link to a WWW page maintained by the manufacturer and describing the particular piece of equipment (if such a page exists) may be included;

2. the basic architecture of the switching fabric (e.g., shared backplane, shared memory, or self-routing; input or output queueing, etc.);

3 . the number of I/O ports and the respective physical layers involved (e.g., $155 \mathrm{Mbps}$ SDH, 34Mbps E-3);

4. the buffering capabilities;

5. details about the network management capabilities;
Detailed lists of the basic information items applying to other categories of components are omitted here, due to lack of space. For full details on the WDB specification see (WATT Proj., 1997b).

WDB employs extensive cross-referencing, implemented as WWW links, between related items (e.g., devices neighbouring in the network topology). Besides the conventional method of following links from within WWW pages, there is an alternative, topology-driven way to access information about a specific device. The "root" page of each testbed provides a link that leads to a graphical representation of the testbed's topology. (For an example, see Figure 2.) Icons are used to represent the various devices, according to their function. A legend provides identification of the device-category associated with each icon. The graphical representation is "active": clicking on the icon representing an item leads to the appropriate location within the WWW page, that hosts the description for this item. Similarly, clicking to an icon in the legend leads to the "table of contents" for the particular device-category associated with this icon. The topology-driven method of access is a particularly suitable way of retrieving information for a set of interconnected devices. On the other 
hand, the ordinary linkage (i.e., from within the flow of text) is more appropriate for an introductory overview of the testbed's capabilities.

\subsection{Information About Research Projects}

The main purpose of the page describing a research project associated with the testbed is to provide a brief profile for the research activity. Typical information items included in the project's description include:

- Identification of the partners involved in the activity, and of any sponsoring parts; in case the project belongs in a formal multi-project research programme (e.g., ACTS), this is so identified.

- The time-frame over which the project is active.

- A statement of the major objectives of the project.

- A statement of the main technical approach.

- A summary of the activity's trial components, with particular emphasis on the usage of the testbed.

- A summary of the expected or realised achievements.

- Links to other information sources for this activity over the WWW, if applicable.

For research projects with a significant trial component, the relevant WDB page also contains a link that points to information about important individual trials. This link leads to the set of trials that belong to the context of this project only. A cumulative list of trials may be accessed from within the testbed's "root" WWW page.

\subsection{Information About Trials}

As already mentioned, the documentation on trials may be accessed from either the "root" page of a testbed (for a cumulative list of all trials relevant to the testbed), or from within the page documenting a particular project associated with the testbed (for the partial list of the trials that belong to the context of this project). In either case, the Web pages documenting trials are organised into the following thematic categories, according to the particular technological issues highlighted by the trials:

- Performance: For trials whose focus is on the quantitative assessment of some performance aspect of a component, subsystem, or the testbed as a whole, under the presence of specified loading conditions.

- Network management: In this case the focus is on all network management issues, including trials on algorithms or devices that perform functions supportive to management. The category includes (but is not limited to) trials on: connection acceptance control; routing; accounting and charging.

- Application and service support: This category relates to the launching and testing of applications and services over the testbed. Trials on user-perceived QoS belong here.

- Interoperability: As the name indicates, the focus here is on interoperability issues, either between the networking platform and an application system, or between distinct networking platforms.

Furthermore, and for all of the trial categories above, individual trials are further classified as performed or planned. As their names indicate, performed trials are accomplished events with finalised results, while planned trials are future events designed and scheduled, but not yet performed. Entries on performed trials are stored in WDB for the record and for future usage of the results produced by the trials. Entries on planned trials are, by their nature, temporary and serve as "posters" announcing the trials-tobe.

The description for a trial, regardless of the trial category and classification, typically includes the following common information:

- An indication of the time when the trial took (performed trials) or will take (planned trials) place.

- Objectives of the trial.

- An identification of key subsystems of the testbed involved in this trial (and links to parts of WDB that include information for these entities).

- Particular actions that were, or will be, required, including testbed setup details and 
timing considerations (e.g., 10 minutes after the commencement of the trial, the load on link $\mathrm{X}$, leading to port $\mathrm{Y}$ of the switch $\mathrm{Z}$, will be increased by $50 \mathrm{Mbps}$ and for the subsequent 30 minutes the losses occurring on switch $\mathrm{Z}$ will be monitored.)

- Expected results, as of the time the trial was designed.

Additionally, the page may include a link to a Java-applet that provides a dynamic demonstration of some aspects of the trial (this feature requires usage of a Java-capable browser); for example, the applet may display an outline of an important time-sequence of procedures or events, or exhibit "typical" fluctuations of measurable quantities, in some appropriate graphical representation. The animation is intended to recapitulate, in an intuitive manner, essential information already supplied by other information items just mentioned.

For trials of "planned" status, typical users of the information described above are persons interested to prepare before actually witnessing the trial. The WDB information provides a suitable briefing so as to enable these users to judiciously focus on interesting elements during the evolution of the trial. If the testbed that will host the planned trial supports WMS, then it is possible to actually "watch" the trial being performed remotely. For this purpose, the page describing the planned trial will include (at some appropriate time before the actual performance of the trial) a link that may be used to invoke the appropriate WMS software and monitor the trial remotely.

In the end, a planned trial will be realised. Accordingly, the classification of the corresponding documentation in WDB will eventually change into "performed" status. Along the process of doing this, the appropriate Web page will be modified, so as to include a description of the trial results (together with a comparison between expected and achieved results) and a comment summarising the conclusions drawn from the trial. Beyond these supplements, the updated page may include links for downloading data files containing material that resulted from the trial and that may be post-processed, or otherwise manipulated by the user, in an offline fashion. For example, a trial that focuses on applications and user-perceived QoS may include audio or moving video files, which, when played back, exhibit deterioration of the QoS under certain trial conditions.

An important special case of such data files applies to testbeds that support WMS: on such a testbed, an operator may record fluctuations of certain aspects of the testbed's status and save this record in an electronic file. As already remarked, in order to generate a dynamic visualisation of the recorded status changes, a user may download and subsequently process this specially formated data file. He can do it locally, at his computer, by means of special software, namely the demonstration authoring tool. As a general rule, data files (of any format) accessible from within the WDB page documenting a performed trial are always accompanied with a commentary on their format, contents, and the possible means for their processing.

\section{Implementation Issues and Operational Status}

A prototype of WDB has been implemented in the framework of the WATT research project. The prototype is complete and operational; it is reachable over the World Wide Web, through the URL: http://www . telecom.ntua.gr/watt $/ w d b /$. The system has been accessed using many different WWW browsers (including recent versions of Netscape Navigator TM and Internet Explorer TM) to ensure that it may be effectively utilised through popular WWW browser software. In general, no special restrictions on the usage of specific browser software exist. Certain features of the system (e.g., invocation of the WMS client) require that the Web browser be Java capable. Nowadays, this can hardly be considered a limitation.

Current initial deployment of WDB covers two major European experimental ATM testbeds; namely EXPERT (the largest broadband test platform in Europe, located in Basel, Switzerland) and AMUSE (a network of 7 broadband centres, located in Italy (Milan), Germany (Munich), Belgium (Mons), Switzerland (Basel), England (Cambridge), Portugal (Aveiro) and Iceland (Reykjavik) and connected through the European ATM network). The EXPERT testbed supports an interface to WMS and thus can be monitored remotely. These two testbeds vary considerably in terms of orientation. The 
EXPERT testbed focuses on Network Management and Network Interoperability issues, while AMUSE aims at multimedia services over a broadband platform. Consequently, the two testbeds are quite different in terms of configuration and setup. The fact that both platforms were able to fit in the WDB structure provides an assurance that the design specification is grounded on a firm basis and that other important ATM testbeds may be incorporated in WDB without a major effort. In fact, it is envisaged that such extensions will indeed occur, as WDB matures. General guidelines for implementing extensions have already been issued by the WATT project (see WATT Proj., 1997b and WATT Proj., 1997f). The initial prototype itself may serve as a concrete implementation paradigm.

The WDB design specification has intentionally left open the option of either using a single central WWW server for storing all the files constituting WDB, or, alternatively, distributing the files into more servers. From the security point of view, the usage of many servers is preferable and the same turns out to be true in terms of ease of maintenance. In fact, the structuring of information suggests that the "information tree" corresponding to a testbed is a "self-contained" unit of information. Using appropriate relative cross-referencing links, an entire tree may be relocated to a different WWW server in a way transparent to the user. The relocation requires only the adjustment of one link pointing to the "root" of the tree-hierarchy.

The WDB prototype has used three WWW servers: one at National Technical University of Athens (NTUA), one by the EXPERT testbed, in Basel, and one by the AMUSE testbed, in Milan. These servers include the following files:

In the NTUA server:

- Introductory pages describing the structure of WDB, various tools, etc.;

- the archives containing the demonstration authoring tool software, available for download; and

- other miscellaneous documents (e.g., WATT deliverables in electronic form).

In the EXPERT server:

- All WWW pages documenting the EXPERT testbed and related projects and trials;
- the WMS client software (to be invoked on top of the user's browser for monitoring the testbed); and

- the WMS server software that must run on that server for WMS to be operational.

In the AMUSE server:

- All WWW pages documenting the AMUSE testbed and related projects and trials.

Most material in WDB is publicly accessible. Some pages, however, contain confidential material, in which case an authentication procedure is initiated when the page is entered. The organisation of the material into multiple WWW servers facilitates the task of providing access classification, in that only servers containing "sensitive" material need to be secure servers, appropriately set-up to enforce restrictions of access. For more details on the security features of WDB, see (WATT Proj., 1997d). (WATT Proj., 1997f) has additional details on implementation issues encountered during the development of the prototype.

\section{Major Technological Experiments Supported by WDB}

Two major experiments/trials supported by WDB and the associated Java-based tools (WMS and the demonstration authoring tool) took place in the scope of large distributed conferences and were witnessed by wide audiences. During these conferences, the Global-360 network (an international IP over ATM platform, configured with the support of National Hosts and Public Network Operators) was used to carry data to several remote sites monitoring the event. Global-360 supported high-bandwidth Internet applications and, in particular, enabled videoconferencing among several sites around the world.

The first trial involved distortion of the quality of a video stream, due to multiplexing with excessive background traffic (Mitrou et al., 1997a). The video stream was transmitted from Basel (the EXPERT's location) to Madeira (one of the locations hosting a 'distributed' conference on network interoperability) over the Global-360 platform. After being routed through a number of ATM switches inside EXPERT, and just 
before leaving the platform, the video stream was multiplexed with background traffic originating from traffic generators. The amount of the background traffic was fluctuating, so as to incur a visible distortion pattern on the video stream. WDB advertised the trial and WMS was used to monitor the bandwidth consumed by the background traffic. In Madeira, the client WMS software, running on top of a Java enabled WWW browser, was operated by a speaker presenting the trial to the conference audience in
Madeira. Additionally, people at other remote sites were given the ability to, either download the client software on their browsers and monitor the trial, or watch the presentation in Madeira through the video-conferencing application. At the same time, the video stream was reproduced on the video-conferencing terminal in Madeira and other sites. The audience could examine distortion of the video signal and associate it with the bandwidth fluctuations that had caused it (see Figure 3).

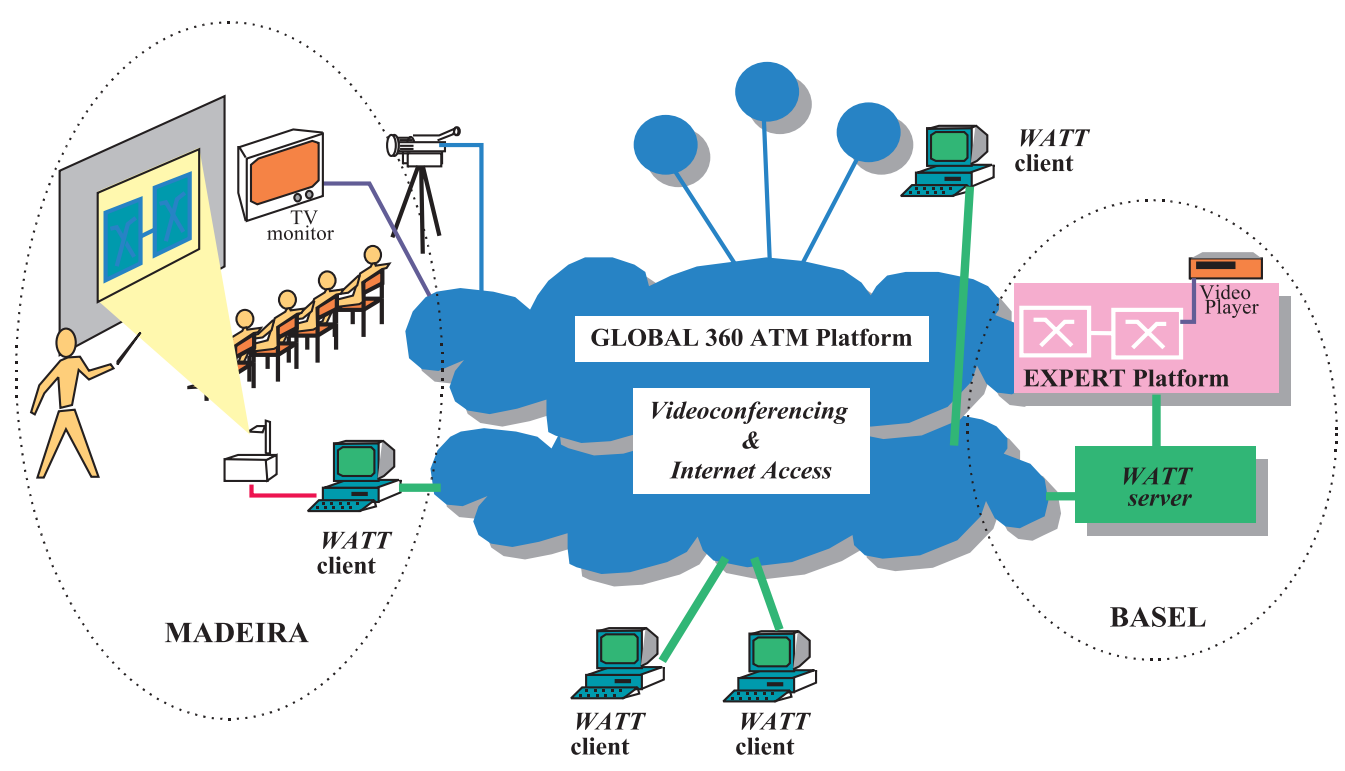

Fig. 3. Remote Monitoring of Background Traffic.

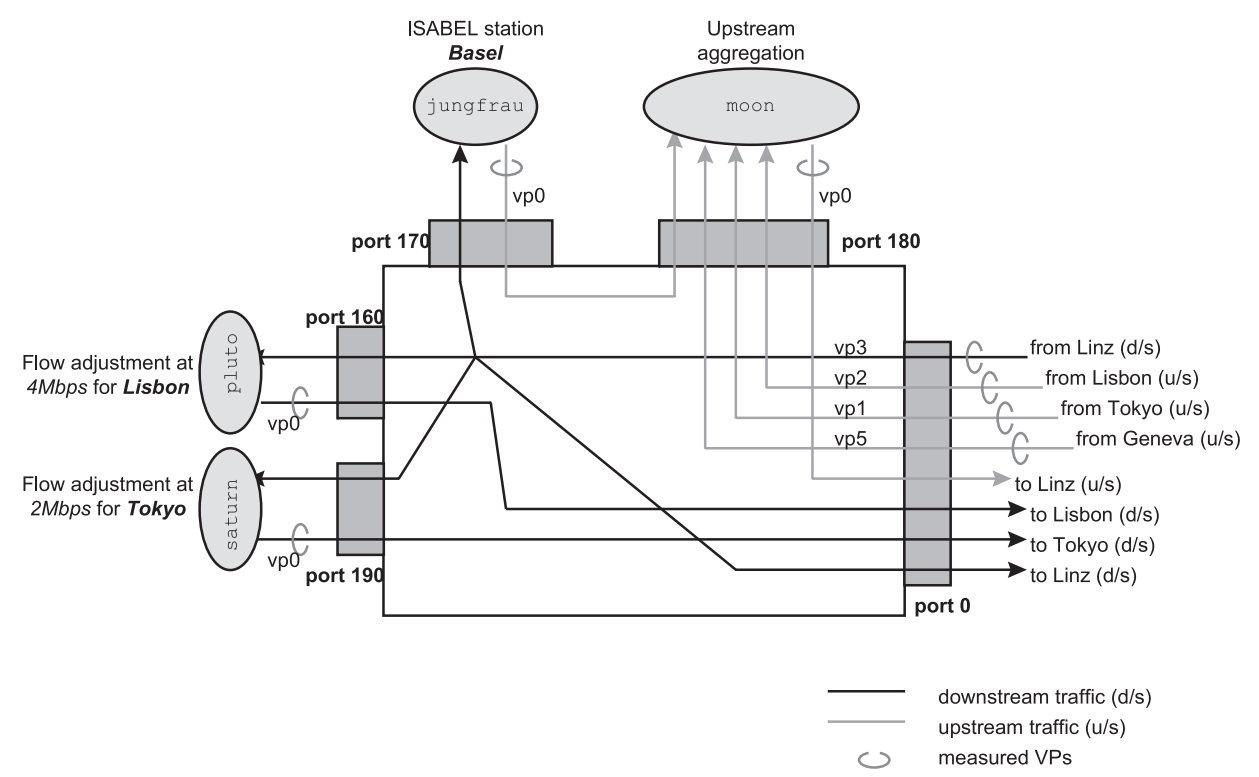

Fig. 4. Remote Monitoring of Bandwidth Consumed in Virtual Paths. 
In the second trial (WATT Proj., 1998), WMS was used to measure the traffic flowing within and outside the EXPERT platform. During this trial, all the connections of Global-360 to the EXPERT platform in Basel passed through a Fore ASX-200 ATM switch. WMS monitored the bandwidth consumed in the various ports of this switch, keeping track of the traffic flow passing through the various connections. The connections used in this experiment are depicted in Figure 4.

\section{Conclusions}

WDB is a Web-based database system that provides information windows (both static and dynamic) for ATM networking platforms. Despite its name, WDB is not a "classical" database, in the sense that it does not rely on specific Database Management Systems (DBMS) or on conventional data structures. Instead, it consists primarily of a set of appropriately organized HTML pages (together with information in other formats, e.g., digitised picture files). Other Java-based tools, accessible through the same WWW environment, provide for on-line monitoring of the testbeds and for the postprocessing of trial results in an off-line fashion. The whole system is readily accessible through an ordinary WWW browser.

In the scope of the WATT project, WDB has been deployed so as to cover two major European experimental ATM platforms, EXPERT and AMUSE. However, WDB has been designed to support a framework much wider than that covering the two immediate target-testbeds, as it is envisaged that organisations, projects, consortia or other entities that possess and/or manage ATM testbeds, not in the direct scope of WATT, will be willing to join WDB and disseminate thereby information about those testbeds and related activities. The design of WDB includes a detailed list of required information items, for all potential information categories, as well as a rigid hierarchical structure that organises the information content. Both the initial development and subsequent updates of the WDB follow these design specifications. The implemented prototype provides a concrete paradigm that may serve as a guideline in future extensions of the database.

\section{References}

[1] A. AlLES, "The Next Generation ATM Switch: From Testbeds to Production Networks", White Paper, 2000, available at http://www.cisco.com /warp/public/c|c/so/neso/vvda/.

[2] L. Chapin AND B. MEANDZIJA, "The Global Internet”, IEEE Transactions Magazine, Vol. 35, No. 5, pp. 50, May 1997.

[3] InfoWin AC113, Technical Annex, March 1996. Information electronically available on the WWW through the URL: http://www. infowin.org/ACTS/RUS/PROJECTS/INFOWIN/

[4] E. LARSON AND B. STEPHENS, Administrating Web Servers, Security, \& Maintenance, Prentice Hall, ISBN 0-13-022534-7, 2000.

[5] J. Magid, R.D. MatThews, AND P. Jones, The Web Server Book. Ventanna Press, 1998.

[6] N. Mitrou, M. PotTs, AND K. KontovasiLis, "Remote Monitoring of an ATM platform through the WATT System", In Proc. 2nd International Distributed Conference on Network Interoperability, Madeira, Portugal, June 1997, available at http://www.telecom.ntua.gr/watt/papers . htm

[7] N. Mitrou, E. Vayias, J. Soldatos, K. KaVIDOPOULOS, G. BRANIS, K. KONTOVASILIS, G. KORMENTZAS, AND C. VADUVA, "Remote Monitoring of Networks through Gateway Programming over the Internet", In Proc. 13th UK Workshop on Performance Engineering of Computers \& Telecom., Ilkley, UK, July 1997, available at http: //www.telecom.ntua.gr/watt/papers . htm

[8] R RAMAKRISHNAN AND J. GEHRKE, Database Management Systems, McGraw Hill, 3rd Edition, 2002.

[9] E. Vayias, J. Soldatos, N. Mitrou, K. KontoVASILIS, AND G. KORMENTZAS, "Managing Networks over the Web: Classification of approaches and an implementation", In Proc. Int. Conference on Telecommunications, Chalkidiki, Greece, June 1998, available at http://www . telecom.ntua.gr/watt/papers.htm

[10] C. Vaduva, E. Vayias, J. Soldatos, N. Mitrou, "Network Information Processing Systems", In Proc. NIPS'97 Conference, Sofia, Bulgaria, October 1997, available at http://www . telecom.ntua.gr/watt/papers.htm

[11] WATT AC235, Technical Annex, March 1996. Information electronically available on the WWW through the URL: http: www.telecom. ntua.gr/watt

[12] WATT AC235, Project Deliverable D05 "Specification of the interface between the database and N\&SM", May, 1997, available at http:// ww.telecom.ntua.gr/watt/deliverables . htm 
[13] WATT AC235, Project Deliverable D02 "Report on the database structure”, May 1997, available at http://www.telecom.ntua.gr/watt/ deliverables.htm

[14] WATT AC235, Project Deliverable D10 “Demonstration authoring tool”, August 1997, available at http://www.telecom.ntua.gr/watt/ deliverables.htm

[15] WATT AC235, Project Deliverable D04 "Security and confidentiality aspects", August 1997, available at http://www.telecom.ntua. gr/watt/deliverables.htm

[16] WATT AC235, Project Deliverable D06, "Interface between the database and N\&SM", November 1997, available at http://www.telecom. ntua.gr/watt/deliverables.htm

[17] WATT AC235, Project Deliverable D03 “Information database structure”, November 1997, available at http://www.telecom.ntua.gr/watt/ deliverables.htm

[18] WATT AC235, Project Deliverable D011, “On Line Demonstrations on Network Interoperability and Multimedia Service Integration", August 1998, available at http://www.telecom. ntua.gr/watt/deliverables.htm

Received: June, 2000 Revised: February, 2003 Accepted: February, 2003

Contact address:

Kimon Kontovasilis George Kormentzas Anastasios Kourtis

National Center for Scientific Research "Demokritos" Institute for Informatics \& Telecommunication

GR-15310 Ag. Paraskevi

POB 60228, Greece

Phone: +302106503130

Fax: +302106532175

e-mail: \{kkont;gkorm;kourtis\}@iit.demokritos.gr

Nikolas Mitrou John Soldatos

Evangelos Vayias

National Technical University of Athens Electrical \& Computer Eng. Dept. Computer Science Division 9 Heroon Polytechneiou str. GR-15773 Zografou, Greece

Phone: +302107721639

Fax: +30 2107722534 e-mail: mitrou@cs.ntua.gr, \{jsoldat; evayias\}@telecom.ntua.gr
KIMON KoNTOVASILIS was born in Athens, Greece on 1964. He received the Diploma in Electrical Engineering from the National Technical University of Athens (NTUA) in 1987, the M.Sc. in Computer Science from the North Carolina State University (NCSU) in 1990 and the Ph.D. in Electrical Engineering from NTUA in 1993. Since 1996 he is with the Institute of Informatics and Telecommunications of the National Centre for Scientific Research "Demokritos" as a member of the research staff, currently ranking as Senior Researcher. His research interests include modelling, performance evaluation, dimensioning and traffic control of broadband wired and wireless telecommunication networks, distributed architectures for network management systems, queueing theory, as well as numerical methods for the solution of Markov Chain models and numerical linear algebra. He has published extensively in the fields above, in international scientific journals, edited books and conference proceedings. Dr. Kontovasilis is a member of IFIP, WG6.3 and the Technical Chamber of Greece.

GEORGE T. KoRMENTZAS was born in Athens, Greece on 1973. He received the Diploma and the Ph.D. in Electrical and Computer Engineering from the National Technical University of Athens (NTUA) in 1995 and 2000 respectively. Since 1996, he is a research associate with the Institute of Informatics \& Telecommunications of the Greek National Center for Scientific Research "Demokritos" working on research projects in the areas of traffic analysis, network control, resource management and quality of service in high-speed wired and wireless networks. He has over fifteen publications in the above fields, in international scientific journals, edited books and conference proceedings. Dr. Kormentzas is a member of WSEAS and the Technical Chamber of Greece.

ANASTASIOS KOURTIS was born in Athens, Greece in 1956. He received his BS degree in Physics in 1978 and his PhD degree in Telecommunications in 1984, both from the University of Athens. From 1986 he has been a member of the research stuff in the Institute of Informatics and Telecommunications of the Greek National Centre for Scientific Research "Demokritos". Currently he is a Senior Researcher. He has worked on encryption systems, digital modulation techniques, spread spectrum systems and multimedia applications. His current research interests are in the areas of broadband wireless networks, Local Microwave Distribution Systems (LMDS) and digital interactive TV.

NiKOLAS M. Mitrou was born in Greece on October 5, 1957. He received the undergraduate Diploma degree in Electrical Engineering from the National Technical University of Athens (NTUA) in 1980, the MSc degree in Systems and Control from the UMIST, Manchester, in 1982 and the PhD degree in Electrical Engineering from NTUA in 1986 In 1988, he joined the NTUA as a Senior Researcher and he is currently a Professor in the Department of Electrical and Computer Engineering. His research interests are in the areas of digital communication systems and signal processing, with emphasis on the architecture, modelling, performance evaluation and optimisation of integrated networks, local area networks and mobile communication systems, digital video and multimedia, having published extensively in these fields. Dr. Mitrou has a leading participation in many research projects (RACE, ESPRIT, STRIDE, ACTS) and he was also the Project Manager of the ACTS WATT project. Prof. Mitrou is a member of the IEEE and the Technical Chamber of Greece and member of IFIP WG 6.3. 
JOHN K. SolDATOS was born in Athens, Greece in 1973. He received the Dipl.Eng. degree from the Department of Electrical and Computer Engineering, National Technical University of Athens, Greece, in 1996. Since 1996, he is a Research Associate with the Telecommunications Laboratory of the Department of Electrical and Computer Engineering of the National Technical University of Athens working on research projects in the areas of Integrated Communication Networks control, management, performance evaluation and WWW based multimedia/hypermedia systems. Mr. Soldatos has had a very active role in a number of European research activities within the ACTS framework (EXPERT AC-094, WATT AC-235), as well as in the ESPRIT 'Chameleon' project. He has also been actively involved in the ACTS IMPACT AC-324 project implementing an ATM resource control and management system using intelligent agents. He is also finishing his $\mathrm{Ph} . \mathrm{D}$. thesis dealing with Traffic Control and Resource Management in Integrated Services Networks. Mr. Soldatos is a student member of the IEEE and a member of the Technical Chamber of Greece.

EVANGELOS K. VAYIAS was born in Athens, Greece in 1973. He received the Dipl. Eng. degree from the Department of Electrical and Computer Engineering, National Technical University of Athens, Greece, in 1996. Since 1996, he is a Research Associate with the Telecommunications Laboratory of the Department of Electrical and Computer Engineering of the National Technical University of Athens, working on research projects in the areas of Integrated Communication Networks control, management and performance evaluation. He has participated in the ACTS EXPERT, NICE and WATT projects and in the ACTS IMPACT project (the latter dealing with the implementation of an ATM resource control and management system using intelligent agents). He is also in the final stage of his Ph.D. thesis dealing with Traffic Control and Resource Management in Computer Networks for end-to-end quantitative QoS. Evangelos Vayias is a member of the Technical Chamber of Greece and student member of the IEEE. 